ISSN: 0213-2087 e-ISSN: 2444-7080

DOI: https://doi.org/10.14201/shhcont382020515

\title{
INTRODUCCIÓN AL DOSIER. NACIÓN Y MASCULINIDADES EN LA ESPAÑA CONTEMPORÁNEA
}

\author{
Xavier Andreu MIRALLES \\ Universitat de València ${ }^{1}$
}

Recibido: 06/07/2020 Aceptado: 20/07/2020

El estudio histórico de las masculinidades, un campo de análisis relativamente reciente, ha abierto nuevas perspectivas para el análisis de los procesos de construcción nacional. La historia de las masculinidades se interroga por la construcción histórica de lo "masculino" y sus consecuencias para la conformación de las subjetividades y para la configuración de las relaciones de poder existentes en una sociedad determinada $^{2}$. Desde este campo de estudio se entiende que el significado de la categoría "masculino» es siempre histórico e inestable, y que para fijarse necesita ser declinada con otras categorías que la cruzan y le confieren sentido: la "feminidad», pero también la etnicidad, la raza, la religión, la clase, etc ${ }^{3}$. La nación es una de ellas. La reflexión es análoga si se enfoca desde el lado contrario. El significado de la nación trata también

1. El autor participa del proyecto de investigación «Narrar el gènere i la nació: Espanya, 1843-1898» (GV2019/111), financiado por la Generalitat Valenciana, y del proyecto «Derechas y nación en época contemporánea. Una perspectiva transnacional» (PGC2018-099956-B-I00), financiado por el Ministerio de Ciencia, Innovación y Universidades del Gobierno de España.

2. Una introducción a este campo de estudio y a algunos de sus conceptos fundamentales en ARESTI, Nerea: "La historia de género y el estudio de las masculinidades. Reflexiones sobre conceptos y métodos". En: Gallego, Henar (ed.): Feminidades y masculinidades en la historiografía de género. Granada: Comares, 2018, pp. 173-194.

3. SINHA, Mrilanini: «Giving Masculinity a History: Some Contributions from the Historiography of Colonial India", Gender \& History, n. 11/3, 1999, pp. 445-460. 
INTRODUCCIÓN AL DOSIER.

NACIÓN Y MASCULINIDADES EN LA ESPAÑA CONTEMPORÁNEA

XAVIER ANDREU MIRALLES

de establecerse en cada contexto histórico a través de su articulación con múltiples categorías, como la de "masculinidad" ${ }^{4}$.

La historiografía española ha presenciado en los últimos años un nuevo interés por la construcción histórica de las masculinidades en el mundo contemporáneo, desde la que se ha abierto la puerta al estudio de cómo éstas se vinculan con las formas de imaginar la nación y con los procesos de construcción nacional ${ }^{5}$. Este dosier monográfico reúne trabajos que se inspiran en estas nuevas tendencias. Aunque parten de planteamientos no siempre coincidentes, todos ellos se interrogan por las formas diversas en que nación y masculinidad se han entrelazado históricamente entre sí y en relación con otras categorías. En este sentido, este dosier no se plantea el análisis evolutivo de las masculinidades, o de las "masculinidades nacionales» en la España contemporánea. Más bien, se pregunta por procesos históricos concretos más generales para cuya comprensión resulta fundamental abordar dicha conexión.

Estos trabajos permiten identificar algunas de las vías de análisis que pueden resultar más fructíferas en el estudio histórico de la relación entre nación y masculinidad en el mundo contemporáneo. En esta introducción hago hincapié en algunas de ellas: el particular vínculo existente entre nación, masculinidad y "modernidad"; la pluralidad de masculinidades nacionales y la pugna por fijar su significado; la dimensión transnacional de estas masculinidades nacionales; la importancia de tener en cuenta el carácter inestable tanto de la nación como de la masculinidad, así como de las relaciones que se establecen entre ambas; o la centralidad del estudio del sujeto y de sus experiencias.

En relación con la cuestión de la «modernidad», la socióloga Raewyn Connell y el historiador George L. Mosse, los grandes pioneros en el estudio de las masculinidades, coincidieron en señalar el cambio trascendental que en la manera de entenderlas se habría producido con la llegada del «mundo moderno». Asimismo, subrayaron que el "hombre moderno" se habría cimentado sobre los discursos nacionalistas propios del mundo contemporáneo ${ }^{6}$. Trabajos más recientes han matizado estas afirmaciones y han puesto de relieve la necesidad de atender a la larga duración, a las continuidades y pervivencias entre las formas de imaginar la nación y de entender la masculinidad antes y después de la era de las revoluciones ${ }^{7}$. Otros autores han subrayado que, a pesar de

4. NAGEL, Joan: "Masculiniy and Nationalism: Gender and Sexuality in the Making of Nations", Ethnic and Racial Studies, 21, 2, 1998, pp. 242-269; HoEgaERTS, Josephine: Masculinity and Nationhood, 1830-1910. Constructions of Identity and Citizenship in Belgium. Nueva York: Palgrave Macmillan, 2014.

5. AResti, Nerea: "La historia de las masculinidades, la otra cara de la historia de género", Ayer, n. 117, 2020, pp. 333-347.

6. Connell, Raewyn: Masculinities. Cambridge: Polity Press, 1995; Mosse, George L.: La imagen del hombre. La creación de la moderna masculinidad. Madrid: Talasa, 2001.

7. Respecto al largo debate sobre la «modernidad» de las naciones, véase la revisión reciente de Moreno, Raúl: "Corrientes teóricas para el estudio de las naciones y el nacionalismo: críticas y alternativas al paradigma modernista", Revista de estudios políticos, n. 171, 2016, pp. 225-253. En relación con las "mascu- 
todo, los procesos revolucionarios liberales y las guerras napoleónicas transformaron radicalmente y al mismo tiempo la forma de entender la nación y la masculinidad ${ }^{8}$.

Por todo ello, el estudio histórico de estas rupturas y continuidades resulta fundamental. En su contribución, Nuria Soriano subraya precisamente la complejidad de las formas diversas de entender la figura del «héroe» en la España ilustrada, nacidas del diálogo con modelos de masculinidad anteriores como los propios del humanismo renacentista o los de un ideal barroco reactualizado y revisado en el siglo XVIII. A su vez, señala la relevancia del «hombre de bien» ilustrado, cuyo heroísmo nacía de su contribución a la felicidad pública y al bienestar de la patria, para entender el reformismo dieciochesco y los modelos de masculinidad patriótica que protagonizaron las revoluciones de finales de aquella centuria. Darina Martykánová y Víctor Núñez, en su artículo sobre la dimensión de género del proceso de construcción de algunas profesiones en la España del siglo XIX, ponen de manifiesto asimismo la pervivencia de elementos procedentes de este mundo ilustrado, que se vieron obligados a transformarse en el contexto revolucionario y sobre la base de la redefinición del «hombre» en tanto que sujeto libre e independiente que llevó a cabo el liberalismo. Estos autores muestran igualmente el carácter dinámico de los ideales de masculinidad. Al ideal ilustrado del hombre de ciencia sensible y útil a la patria, le sucedió un ideal romántico que añadió como elemento central el amor a la humanidad. A su vez, este modelo fue cuestionado en las últimas décadas del siglo XIX por una visión más positivista y desapasionada de lo que debía ser la función pública. No obstante, como señalan Martykánová y Núñez, estos ideales diversos no fueron estancos o consecutivos, sino que se solaparon unos con otros a lo largo del siglo XIX.

Con todo, más allá de este debate necesario sobre el cambio y las continuidades que supusieron la llegada del mundo contemporáneo, resulta interesante analizar cómo nación y masculinidad se conjugaron con la propia categoría de «modernidad». Entendido como una serie de indicadores «objetivos» a alcanzar, este concepto encierra una visión normativa del desarrollo de las sociedades que sanciona o legitima las

linidades», Harvey, Karen y SHePard, Alexandra: "What Have Historians Done with Masculinity? Reflections on Five Centuries of British History, circa 1500-1950", Journal of British Studies, n. 44, 2005, pp. 274-280; French, Henry y Rothery, Mark: "Hegemonic Masculinities? Assessing Change and Processes of Change in Elite Masculinity, 1700-1900». En: ArNold, John H. y Grady, Sean (eds.): What is Masculinity? Historical Dynamics from Antiquity to the Contemporary World. Basingstoke: Palgrave Macmillan, 2013, pp. 139-166.

8. Hagemann, Karen: "Of 'Manly Valor' and 'German Honor': Nation, War, and Masculinity in the Age of the Prussian Uprising against Napoleon", Central European Review, n. 30/2, 1997, pp. 187-220; DuDiNK, Stefan, Hagemann, Karen y Tosh, John (eds.): Masculinities in Politics and War. Gendering Modern History. Manchester y Nueva York: Manchester University Press, 2008. Respecto a la transformación cualitativa y cuantitativa del concepto de nación en el periodo revolucionario véase SEwell JR., William H.: "The French Revolution and the Emergence of the Nation Form». En: Morrison, Michael y Zook, Melinda (eds.): Revolutionary Currents: Nation Building in the Transatlantic World, 1688-1821. Nueva York: Rowman and Littlefield, 2004, pp. 91-125. 
INTRODUCCIÓN AL DOSIER.

NACIÓN Y MASCULINIDADES EN LA ESPAÑA CONTEMPORÁNEA

XAVIER ANDREU MIRALLES

relaciones de poder que se establecen entre ellas". Sin embargo, la «modernidad» entendida como uno de los prismas fundamentales a través de los cuales se ha pensado el mundo contemporáneo, resulta trascendental para entender la relación entre nación y masculinidad. En el momento en el que las naciones empiezan a imaginarse históricamente, la «modernidad» de los sujetos que las integran deviene un indicador de la modernidad de la nación en su conjunto y en una de las herramientas legitimadoras de las relaciones de poder que se establecen entre las diferentes naciones y en el seno de cada una de ellas.

Aunque el proceso se ha estudiado principalmente en relación con las mujeres, afectó igualmente a los discursos sobre la masculinidad, como ya sostuvo George L. Mosse $^{10}$. Los trabajos reunidos en este dosier coinciden en la relevancia de esta conexión. El «héroe ilustrado» analizado por Nuria Soriano fue pensado como un hombre "civilizado" y moderno, capaz de regular y dirigir sus pasiones hacia el bien común de la patria frente al héroe guerrero y feroz de épocas anteriores, que tuvo que ser «domesticado». El proceso se observa particularmente a través de la relectura que llevaron a cabo los ilustrados españoles de los conquistadores americanos. El eje civilizaciónbarbarie fue fundamental también para médicos e ingenieros de la época romántica. Para conseguir su consolidación y reconocimiento, estos profesionales se presentaron como la encarnación de un proyecto civilizatorio identificado con el liberalismo y con la modernidad y asociaron su suerte a la de la nación española en su conjunto.

El eje civilización-barbarie o modernidad-atraso fue particularmente relevante, a la hora de fijar y prescribir los ideales de masculinidad, a finales del siglo XIX, en el contexto de los debates sobre la degeneración racial y nacional de la era del imperialismo ${ }^{11}$. Tras el Desastre del 98, las supuestas carencias civilizatorias de España y de los españoles se acentuaron hasta el punto de provocar una "crisis de masculinidad nacional. En su artículo, David Castro aborda originalmente esta cuestión a través del análisis de las consecuencias del Desastre en un ámbito claramente identificado

9. Chakrabarty, Dipesh: Al margen de Europa: pensamiento poscolonial y diferencia histórica. Barcelona: Tusquets, 2008.

10. Mosse, George L.: Nationalism and Sexuality. Respectability and Abnormal Sexuality in Modern Europe. Nueva York: Howard Fretig, 1985.

11. Esta cuestión ha sido ampliamente estudiada para el caso español; AREsTi, Nerea: "A la nación por la masculinidad. Una mirada de género a la crisis del 98”. En NASH, Mary (ed.): Feminidades y masculinidades. Arquetipos y prácticas de género. Madrid: Alianza Editorial, 2014, pp. 47-74; Aresti, Nerea, PETERS, Karin y BRÜHNE, Julia (coords.): ¿La España invertebrada? Masculinidad y nación a comienzos del siglo XX. Granada: Comares, 2016; ArEsti, Nerea y MARTYKÁNová, Darina (coords.): «Masculinidades, nación y civilización en la España contemporánea", Cuadernos de historia contemporánea, n. 39, 2017; ZABALGOITIA, Mauricio (coord.): Hombres en peligro. Género, nación e imperio en la España de cambio de siglo (XIX-XX). Madrid: Iberoamericana-Vervuert, 2017; TORRes, Gemma: La virilitat d'Espanya a l'Àfrica. Nació $i$ masculinitat al colonialisme al Marroc (1880-1927). Catarroja: Afers, 2020. 
con lo español como el toreo. Para la mayor parte de los autores regeneracionistas, el matador se convirtió en epítome de una barbarie española que era necesario erradicar. No obstante, la generación del 14 planteó otra vía de regeneración de la masculinidad nacional mediante una idealización del diestro Juan Belmonte que «salvaba» al espectáculo taurino de sus críticas y lo hacía compatible con la «modernidad».

El peso de la «modernidad» es perceptible también en la «vergüenza» sentida por aquellos españoles que lamentaban la incapacidad de sus compatriotas para estar a la altura de lo que se exigía de ellos y sostener con su esfuerzo la empresa imperial africana, como estudia Gemma Torres en su artículo sobre las emociones viriles que se pusieron en juego en el contexto de las Guerras del Rif (1909-1927). Está presente también en la búsqueda de una modernidad fascista que superara a una anti-España fofa y vulgar, encarnada en una determinada manera de entender y festejar las Fallas valencianas, como señala Zira Box en su artículo. El debate sobre la modernidad de la nación, y su dimensión de género, siguen siendo centrales en el tiempo presente, como apunta Sara Santamaría en su colaboración. Santamaría analiza relatos recientes de viajeros o residentes españoles que narran su paso por Guinea Ecuatorial, la antigua colonia española. La manera que tienen estos autores de aproximarse al país africano descansa en un concepto de modernidad que, reformulado a través de teorías como la del desarrollo o de concepciones neoliberales del sujeto moderno que ponen el énfasis en su "libertad" sexual, sanciona las relaciones de poder entre el mundo occidental y sus antiguas colonias en un contexto postimperial.

En segundo lugar, muchos de los trabajos de este dosier ponen de manifiesto la utilidad del concepto de "masculinidad nacional», tras despojarlo por supuesto de todo esencialismo. Entendida como un ideal al que se atribuyen en un contexto histórico concreto los valores y las esencias nacionales, la "masculinidad nacional" tiene una dimensión política evidente, con efectos muy relevantes sobre la vida de quienes son animados o requeridos a identificarse con ella. En todo caso, como demuestran los diversos textos aquí reunidos, no puede hablarse de «una» masculinidad nacional, sino de la pugna que mantienen diversos actores históricos por definirla. Una pugna que, en ocasiones, resulta especialmente relevante, como en aquellos momentos en los que se vive una "crisis de masculinidad nacional», cuando por diversas circunstancias se considera que se está produciendo una fractura en el ideal de masculinidad hegemónico ${ }^{12}$. David Castro explora esta cuestión en su texto sobre los efectos del Desastre del 98 sobre la forma de entender e interpretar el toreo. En su trabajo, Gemma Torres analiza otro de estos episodios críticos, acaecido en España tras las derrotas militares sufridas por los ejércitos españoles en el norte de África en las primeras décadas del siglo XX. Torres identifica tres ideales de masculinidad nacional entonces en disputa:

12. Kimmel, Michael: Manhood in America. A Cultural History. Nueva York: Oxford University Press, 1996. 
los propuestos por la generación de militares antiliberales que vieron en el imperio africano el mejor medio de revertir la degeneración de España; los de quienes también confiaban en el imperio como una forma de redención, aunque desde un planteamiento liberal inspirado en la idea de su "misión civilizadora»; y los de aquellas culturas políticas obreras o catalanistas que vertebraron una crítica anticolonial que incluía también una discusión de aquellos ideales de masculinidad nacional que eran asociados al proyecto imperialista.

Estas «masculinidades nacionales» requieren de unos Otros frente a los que definirse $^{13}$. Zira Box analiza en su artículo, por ejemplo, cómo tras la Guerra Civil, el nuevo Estado franquista se propuso la transformación de la fiesta de las Fallas como parte de un proyecto más amplio encaminado a la redefinición y virilización de la nación española. Para este proyecto, las Fallas republicanas se convirtieron en una representación de la Anti-España afeminada que debía ser purificada. La nación viril (fuerte, lineal, austera) que trataban de erigir los franquistas en sus Fallas de la Victoria era perfilada mediante su contraposición con una España vulgar, blanda y a la vez grotesca que era considerada la propia del periodo republicano. La feminidad no es la única categoría a través de la cuál intenta fijarse el significado de esta "masculinidad nacional». Otras categorías como la raza y la sexualidad, que exploran Gemma Torres o Sara Santamaría en sus trabajos, pueden ser en determinados contextos igual o más relevantes. La clase puede ser también una variable fundamental, como se desprende del texto de Darina Martykánová y Víctor Núñez. Los ingenieros y médicos españoles de la época romántica se construyeron como representantes de una masculinidad liberal respetable excluyendo (o subordinando) a las mujeres de sus profesiones. No obstante, lo hicieron también a través de su distanciamiento respecto a otros varones pertenecientes a profesiones consideradas incivilizadas (manuales) o afeminadas (profesiones liberales "poco útiles»).

A pesar de la pluralidad de las «masculinidades nacionales» — o de los proyectos de masculinidad nacional - es fundamental atender a aquéllas que devienen hegemónicas en un momento histórico determinado. En su artículo, Iker González-Allende sostiene

13. MсCunTock, Anne: Imperial Leather: Race, Gender and Sexuality in the Colonial Contest. Nueva York y Londres: Routledge, 1995; SINHA, Mrilanini: Colonial Masculinity. The 'manly Englishman' and the 'effeminate Bengali' in the late nineteenth century. Manchester y Nueva York: Manchester University Press, 1995; MAYER, Tamar (ed.): Gender Ironies of Nationalism. Sexing the Nation. Londres y Nueva York: Routledge, 2000; SinHA, Mrilanini: «Nations in an Imperial Crucible». En: Levine, Phillippa (ed.): Gender and Empire. Oxford: Oxford University Press, 2004, pp. 181-202; TosH, John: "Hegemonic Masculinity and the History of Gender». En: Dudink, Stefan, Hagemann, Karen y Tosh, John (eds.): Masculinities in Politics and War. Gendering Modern History. Manchester y Nueva York: Manchester University Press, 2008, pp. 41-58; DorLIN, Elsa: La matrice de la race. Généalogie sexuelle et coloniale de la Nation française. París: La Découverte, 2009; Ellis, Heather y Meyer, Jessica (eds.): Masculinity and the Other: Historical Perspectives. Newcastle upon Tyne: Cambridge Scholars Publishing, 2009; Torres, Gemma (coord.): "L'home espanyol davant dels seus altres: masculinitat, colonialitat i classe», Rubrica contemporanea, n. 7/13, 2018. 
que durante el franquismo fue hegemónico entre exiliados y emigrados un modelo de masculinidad nacional "cívica" (definida por el trabajo, la paternidad y la diferencia clara entre los papeles a desempeñar entre hombres y mujeres) que afectó a la manera que tuvieron de vivir su masculinidad. A su vez, como explora Gemma Torres en su apartado sobre los discursos anticoloniales de los años veinte y treinta del siglo XX, toda hegemonía es siempre contestada y aquellas concepciones de la masculinidad que son marginadas del proyecto nacional hegemónico en un momento dado pueden acabar modificando o subvirtiendo dicha hegemonía ${ }^{14}$.

En tercer lugar, los textos aquí reunidos ponen de manifiesto la dimensión transnacional de estas masculinidades nacionales. Los ideales de masculinidad, como las naciones, se configuran siempre en la interacción de dinámicas locales, regionales y globales ${ }^{15}$. Nuria Soriano, por ejemplo, señala cómo el ideal patriota del héroe ilustrado se enmarcó en un proceso europeo más amplio de redefinición de la masculinidad en el contexto de la difusión de los nuevos discursos sobre la civilización de las costumbres. Darina Martykánová y Víctor Núñez plantean esto mismo para una cronología posterior, la de mediados del siglo XIX. Sara Santamaría, por su parte, analiza los discursos sobre la masculinidad española postimperial en el marco de la generalización de un ideal de hombre neoliberal de alcance global.

Determinadas dinámicas transnacionales, como los movimientos migratorios, pueden resultar decisivas en la conformación de las naciones ${ }^{16}$. Otro tanto ocurre respecto a las masculinidades. Esta cuestión es fundamental en la contribución de Iker González-Allende sobre el exilio y la emigración en la España franquista. Este autor estudia de forma comparada los efectos sobre las masculinidades del exilio republicano a México posterior a la Guerra Civil y de la emigración económica hacia Alemania de las décadas de 1960 y 1970. A pesar de las grandes y lógicas diferencias entre ambos procesos, así como de su enorme complejidad, González-Allende pone de manifiesto cómo los mecanismos de adaptación a la nueva realidad y la manera que tuvieron de vivir su «masculinidad nacional» estos hombres españoles «desplazados» siguieron unas pautas similares.

14. Domínguez, Pablo y Wendt, Simon (eds.): Masculinities and the Nation in the Modern World. Between Hegemony and Marginalization. Nueva York: Palgrave Macmillan, 2015.

15. Connell, Raewyn y Messerschmidt, James W.: "Hegemonic Masculinity. Rethinking the Concept", Gender E Society, n. 19/6, 2005, pp. 829-859; STORM, Eric: "The Spatial Turn and the History of Nationalism: Nationalism between Regionalism and Transnational Approaches». En: Berger, Stefan y STORM, Eric (eds.): Writing the History of Nationalism. Londres: Bloomsbury, 2019, pp. 215-238.

16. ConRAD, Sebastian: Globalisation and the Nation in Imperial Germany. Cambridge: Cambridge University Press, 2006. 
INTRODUCCIÓN AL DOSIER.

La dimensión transnacional de las "masculinidades nacionales» está inscrita además en su propia naturaleza, como lo está en la de todas las naciones ${ }^{17}$. Para ser fijado, su significado necesita de aquellas otras masculinidades "no nacionales» respecto a las que se define. El peso de la mirada externa resulta en ocasiones decisivo. Como señala Nuria Soriano, en la redefinición de los «héroes ilustrados» españoles cumplió un papel muy relevante la reacción ante una concepción europea de España y de sus habitantes que los convertía en la esencia del atraso y de la decadencia. Los jesuitas expulsos que se destacaron por su labor apologética respondían a estos ataques apelando a la adecuación de sus compatriotas al discurso sobre la modernidad europea. Asimismo, el ideal de "masculinidad nacional» encarnado según los autores de la Generación de 1914 por el torero Juan Belmonte, que analiza David Castro, se presentaba como genuinamente español e inspirado a su vez en rasgos propios del gentleman inglés. El dolor y la vergüenza sentidos frente a otras naciones europeas y la voluntad de ejercer su función civilizadora a la par que ellas, fue fundamental en la redefinición de la masculinidad nacional y en los proyectos imperialistas sobre el Norte de África, como ha estudiado en profundidad Gemma Torres. Esta autora ha demostrado cómo esta masculinidad nacional imperial se construyó especularmente en relación con otras masculinidades coloniales a través de las cuáles era definida ${ }^{18}$. Unas dinámicas que detecta también Sara Santamaría en su análisis sobre la representación del Otro guineano en la España reciente.

En cuarto lugar, en los textos aquí reunidos se observa una voluntad de abandonar una concepción excesivamente estable y rígida de la relación que se establece entre nación y masculinidad en el mundo contemporáneo. Una de las grandes aportaciones de la crítica feminista a la teoría de las naciones ha sido señalar cómo el nacionalismo es una pieza fundamental en la construcción contemporánea de la diferencia sexual. Ésta funciona como una de las llaves maestras con las que legitimar el gobierno de las diferencias entre las diversas naciones y también en el seno de la comunidad nacional ${ }^{19}$. Nira Yuval-Davis ha establecido las funciones y espacios diferenciados que prescriben los nacionalismos contemporáneos a los hombres y a las mujeres. A estas últimas se les limita la capacidad de acción dentro de los movimientos nacionalistas y se las convierte en sujetos pasivos, cuyos cometidos principales pasan a ser los de símbolo

17. Anderson, Benedict: The Spectre of Comparisons: Nationalism, Southeast Asia and the World. Londres: Verso, 1998; Thiesse, Anne-Marie: «National Identities: a Transnational Paradigm». En: DiecKHoff, Alain y JAFFrelot, Christophe (eds.): Revisiting Nationalism. Theories and Processes. Londres: Hurst \& Company, 2005, pp. 122-143; Joep LeERsSEN: El pensament nacional a Europa. Catarroja: Afers, 2019.

18. TORRES, Gemma: La virilitat d'Espanya a l'Àfrica.

19. McCuntock, Anne: Imperial Leather; Blom, Ida, Hagemann, Karen y Hall, Catherine (eds.): Gendered Nations. Nationalisms and Gender Order in the Long Nineteenth Century. Oxford y Nueva York: Berg, 2000. Una revisión reciente de estos debates en ANDREU, Xavier: «El género de las naciones. Un balance y cuatro propuestas", Ayer, n. 106, 2017, pp. 21-46. 
de la nación, sus reproductoras biológicas y culturales y las guardianas de su honor ${ }^{20}$. Los hombres, por su parte, suelen ejercer en el nacionalismo un papel activo en tanto que protagonistas de una comunidad política basada en la camaradería horizontal y en rasgos como la dominación, la violencia o la guerra ${ }^{21}$.

Ahora bien, esta manera de entender la relación entre género y nación en el mundo contemporáneo debe contemplarse siempre como el resultado de procesos históricos, evitando reforzar una comprensión demasiado rígida de dicha relación que acabe naturalizando la diferencia sexual. Si no existen ni una feminidad ni una masculinidad esenciales, derivadas de la naturaleza biológica de los cuerpos, éstas tampoco se articulan siempre del mismo modo con el nacionalismo o las identidades nacionales. No existe, por ejemplo, una única manera de entender las relaciones familiares y de utilizarlas como metáforas de la comunidad nacional. Por otro lado, si bien la nación ha tendido a ser caracterizada con rostro de mujer, no siempre lo ha sido de ese modo ${ }^{22}$. Tanto el africanismo antiliberal que analiza Gemma Torres, como los franquistas que estudia Zira Box, imaginaron a España a través de metáforas sobre su virilidad. Una virilidad que podía encarnarse también en cuerpos de mujer o en ciudades (como la Valencia de postguerra) imaginadas como femeninas, como subraya Box en su contribución.

En un sentido similar, si bien en el mundo contemporáneo lo masculino se ha asociado más habitualmente con el nacionalismo, la guerra o la política, tal asociación no es resultado de la supuesta existencia de una esencia masculina que hace a los hombres más propensos a tomar las armas u ocupar el espacio público, sino un producto de la historia ${ }^{23}$. El análisis de los relatos anticoloniales que propone Gemma Torres o el de las vivencias narradas por unos españoles que experimentan su exilio o su emigración como su particular derrota, que lleva a cabo González-Allende, muestran cómo existen siempre modelos de masculinidad que rechazan esa identificación, por lo que no debe naturalizarse. Las reflexiones de Darina Marykánová y Víctor Núñez sobre la importancia de la sensibilidad y el amor a la humanidad en la masculinidad romántica del siglo XIX plantean igualmente la necesidad de no establecer apriorismos a la hora de abordar los rasgos que definieron lo «masculino» en las sociedades del pasado.

Estas precauciones son necesarias también respecto a la sexualidad. Como han señalado diversos autores, en el mundo contemporáneo el nexo entre el nacionalismo, la masculinidad y lo heteronormativo han resultado hegemónicos. Ahora bien, lo han

20. Yuval-Davis, Nira: Gender E Nation. Londres: Sage, 1997.

21. NAGEL, Joan: "Masculinity and Nationalism».

22. LaKe, Marilyn: "The Ambiguities for Feminists of National Belonging: Race and Gender in the Imagined Australian Community", en Blom, Ida, Hagemann, Karen y Hall, Catherine (eds.): Gendered Nations, pp. 159-176; ARESTI, Nerea: «De heroínas viriles a madres de la patria. Las mujeres y el nacionalismo vasco (1893-1937) ", Historia y política, n. 31, 2014, pp. 281-308.

23. Bracewell, Wendy: "Rape in Kosovo: Masculinity and Serbian Nationalism", Nations and Nationalism, n. 6/4, 2000, pp. 563-590. 
sido como consecuencia de procesos históricos que deben ser explicados, pues dicha conexión no es natural —ni resulta, por ello, necesaria ${ }^{24}$. En el presente neoliberal, como señala Sara Santamaría a propósito de los relatos del diplomático español Luis Melgar, el nacionalismo es compatible con determinados modelos homonormativos que permiten sancionar, por ejemplo, la «modernidad» española frente a sus antiguas posesiones coloniales u otros lugares del planeta. Estos "homonacionalismos» sirven, en este sentido, para legitimar relaciones desiguales de poder a escala global.

Finalmente, muchos de los trabajos reunidos en este volumen monográfico apuntan a la necesidad de situar a los sujetos históricos en el centro del análisis en el estudio de unas masculinidades siempre fragmentadas e inestables ${ }^{25}$. En este sentido, coinciden con aquellos trabajos recientes que, desde la teoría de las naciones y de los nacionalismos, han reclamado la atención sobre cómo «experimentan» la nación los sujetos históricos y cómo participan activamente de su producción y reproducción ${ }^{26}$. Unas «experiencias» al mismo tiempo nacionales y de género que, como señala Gemma Torres, se producen principalmente a través de las emociones, que resultan fundamentales para el estudio de estos procesos. Igualmente centrales son unos sujetos que, a pesar de sus limitaciones, disponen siempre de capacidad de acción: aprovechando los discursos existentes sobre la civilización y la respetabilidad para negociar a través de ellos su posición social entre las elites liberales y ser reconocidos y actuar como hombres públicos, como en el caso de los médicos e ingenieros españoles del siglo XIX; contribuyendo con sus acciones a la construcción simbólica de sí mismos como paradigmas de una determinada manera de entender la masculinidad nacional, como hizo el torero Juan Belmonte; discutiendo con su pluma los discursos imperialistas hegemónicos y sus modelos de masculinidad nacional, como hicieron Ramón J. Sender y otros escritores obreristas en las primeras décadas del siglo XX; o resistiendo los intentos oficiales de «virilizar» y domesticar las Fallas desde una cultura festiva valenciana cuyo control pleno escapó siempre de las manos de las autoridades franquistas.

24. Mosse, George L.: Nationalism and Sexuality; MaYer, Tamar (ed.): Gender Ironies of Nationalism; LANDES, Joan B.: Visualizing the Nation. Gender, Representation, and Revolution in Eighteenth-Century France. Ithaca y Londres: Cornell University Press, 2001; PUAR, Jasbir K.: Ensamblajes terroristas. El homonacionalismo en tiempos queer. Barcelona: Bellaterra, 2017.

25. Wetherell, Margaret y EdLey, Nigel: «Negotiating Hegemonic Masculinity: Imaginary Positions and Psycho-Discursive Practices», Feminism E Psychology, n. 9/3, 1999, pp. 335-356; WhiteHEad, Stephen: «Hegemonic Masculinity Revisited", Gender, Work and Organization, n. 6/1, 1999, pp. 58-62; ConNell, Raewyn y Messerchsmidt, James W.: "Hegemonic Masculinity».

26. ARChilÉs, Ferran: «Lenguajes de nación. Las 'experiencias de nación’ y los procesos de nacionalización: propuestas para un debate», Ayer, n. 90, 2013, pp. 91-114; Molina, Fernando: «La nación desde abajo. Nacionalización, individuo e identidad nacional», Ayer, n. 90, 2013, pp. 39-63; Moreno, Raúl: "La nación de los sujetos: propuestas para una investigación de los fenómenos nacionales a comienzos de la época contemporánea", Rubrica contemporánea, n. 6/11, 2017, pp. 5-23. 
En definitiva, todos estos trabajos al estudiar cómo se articularon las categorías de nación y masculinidad en contextos específicos de la España contemporánea, arrojan nueva luz a la comprensión de procesos históricos de gran calado como el reformismo borbónico dieciochesco, la construcción de una ciudadanía liberal respetable en el siglo XIX, la difusión y popularización de nuevas identidades sociales con la llegada de la cultura de masas, el imperialismo africanista y sus resistencias, la reordenación cultural y simbólica que siguió al triunfo del franquismo y sus límites, la experiencia de la emigración y del exilio en la segunda mitad del siglo XX o los discursos neocoloniales y las ansias de modernidad que siguen presentes en las formas de imaginar la nación española en las primeras décadas de la presente centuria. 